\title{
STUDY ON SUITABILITY EVALUATION MODEL OF NEW MAIZE VARIETIES
}

\author{
Weili Wang, Xiaodong Zhang*', Xiao He \\ College of Information and Electrical Engineering, China Agricultural University, Beijing, \\ China, 100083 \\ * Corresponding author, Address: College of Information and Electrical Engineering, China \\ Agricultural University, 17 Tsinghua East Road, Beijing, 100083, P. R. China, Tel: +86-10- \\ 62737628, Email: zhangxd@cau.edu.cn
}

Abstract: $\quad$ Seed industry is an important basic industry in agricultural, an emerging industry as well. However, the new varieties are driving force and providing source for seed industry development. How extending the new varieties to the most appropriate place are important issues that seed industry needs to address. East of North China as an example, the county for the evaluation unit, on the basis of average daily meteorological data of all meteorological stations in the 50 years and the test data of new varieties of maize in the district, this paper discussed the suitability evaluation model of new maize varieties based on the characteristic values of new maize varieties (temperature, plant diseases and insect pests, lodging and yield) and the corresponding characteristic values of the environment; besides, quantifies the relationship between all characteristic values and their effect on yield; finally, recommends the region that new maize varieties suit and the varieties that fit for regions, providing the premise basis for maize varieties matching.

Keywords: new maize varieties suitability evaluation

\section{INTRODUCTION}

Seed is the most basic, indispensable and irreplaceable agricultural production and the internal of yield, the carrier of various production technologies as well. Therefore, requirements of improved varieties greatly 
exceed other productions. Especially the high-yield, high-quality and highefficiency agriculture establishing, farmers urgently call for stronger competitiveness of the varieties. The dominant of seed production is new varieties. New varieties can be used in production, transformed into real productive forces after regional test, validation and promotion. Vigorously promoting the excellent new varieties of crops is to promote the development of agricultural technology in the most effective measures and the important guarantee.

At present, as a member of WTO, There are higher demands on the corn seed industry development because of international economic integration in China. Promotion of new varieties of maize, as the focus of seed industry development, we must make improvements. However, the current agricultural production, cultivation of maize, some did not fully take into account the suitability and stability, blindly promoted based on past experience and resulted in undue losses. So, we need to create a best suitability assessment model of maize varieties, quantitative calculate the extent appropriate, reduce the choice to promotion region blindness and subjectivity and the promoting risk, improve the average corn yield, and adapt seed market for the new competitive situation.

\section{STUDIES ON SUITABILITY EVALUATION MODEL OF NEW MAIZE VARIETIES}

New maize varieties suitability evaluation can be divided into two types: (1) Given a new maize variety, study its suitability for promotion of the region and quantify the appropriate extent. (2) Given a study area, determine which new varieties of maize in the region fit, while which not, quantify the appropriate extent as well. The appropriate level will be measured by the average yield per acre. The second method was adopted in the paper, with east of north China as the study area.

\subsection{Determining evaluation factors}

There are many factors in judging whether a new maize variety suits the study area. Including climate, pests, soil and so on (Liao, 2002; Li, 2002; Liu, 2003; Huang, 2004; Zhang, 2006). This paper mainly discussed the accumulated temperature (Yu, 1997; Wang, 1997), pests and lodging that will impact on the suitability. Thus, evaluation factors include: temperature $\left({ }^{\circ} \mathrm{C}\right)$, big spot disease(level), small spot disease(level), bending fungus(level), 
gray leaf spot disease(rank), black silk(\%), smut(\%), stem $\operatorname{rot}(\%)$, sheath blight(level), MDM(level), rough dwarf virus(\%), corn borer(\%), lodging rate $(\%)$ Weights of each evaluation factor.

The first condition that new maize varieties promotion should be satisfied with is that no less than $10^{\circ} \mathrm{C}$ environmental temperature will meet the required temperature during the growth period. Once the environmental temperature does not meet, $100 \%$ losses will be resulted in. Therefore, the counties can not participate in suitability evaluation if the varieties are not met all the required temperature in growth period in the counties. Since the suitability evaluation is carrying out according to the yield per acre in the end, the weights of each factor are determined by the extent affecting on the yield, using $\mathrm{V}$ to stand for.

Kinds of diseases and pests and lodging in the event of a serious will cause the production and the degree of reduction not the same. Put the loss of production rate caused by the most serious reduction as weight of a factor. Such as, big spot in the event of a serious disease in the year, susceptible output maybe about $50 \%$. Thus, put $50 \%$ as a big stain on the weight. By analogy, all factors affecting the weight, as shown in table 1:

Table 1. Factors and corresponding weights

\begin{tabular}{|c|c|c|c|c|c|}
\hline Factors & Temperature & Big spot & Small spot & Bending fungus & Gray spot \\
\hline Weight (V) & 1 & 0.5 & 0.6 & 0.6 & 0.5 \\
\hline Factors & Black silk & Corn borer & smut & Stem rot & Sheath blight \\
\hline Weight (V) & 0.7 & 0.2 & 0.3 & 0.5 & 0.2 \\
\hline Factors & MDM & Rough dwarf virus & lodging & & \\
\hline Weight (V) & 0.1 & 0.5 & 0.4 & & \\
\hline
\end{tabular}

\subsection{Establishing the evaluation factor membership function}

\subsubsection{Accumulated temperature membership function}

According to the required accumulated temperature under new maize varieties growing period and counties no less than $10^{\circ} \mathrm{C}$ accumulated data in east of north China. Based on the following formula we can calculate temperature suitability of a certain new variety

$$
S=\frac{T_{\text {sum }}^{*}}{T_{\text {sum }}}
$$


$T_{\text {sum }}$ is the no less than $10^{\circ} \mathrm{C}$ accumulated temperature of region in east of north China. $T_{\text {sum }}^{*}$ is the required accumulated temperature under growing period. Under the appropriate size, the results will be divided into appropriate and inappropriate. Classification as shown in table 2:

Table 2. Suitability classification

\begin{tabular}{llc}
\hline Level & & Suitability \\
\hline One grade & (inappropriate) & $>1$ \\
Two grade & (appropriate) & $\leq 1$ \\
\hline
\end{tabular}

$\mathrm{S}>1$, illustrates that the provided temperature does not satisfy with the required data, so it should not promote in this environment. While $S \leq 1$, is on the contrary. However, the value of suitability degree is not the same. The value of production was affected by the difference in size. We set the true value of $S$ when $S \in[0.9,1.0]$, while 0.9 when $S<0.9$. This is because when $S \in[0.9,1.0]$, environmental temperature can be sufficient for maize varieties growth period. When the large accumulated to a certain extent, more temperature does not engage in other activities in the agricultural. Thus, a formula can be expressed in quantitative the effect upon yield as follow:

$$
T=1-S\left\{\begin{array}{l}
\mathrm{S} \text { true value, } \mathrm{S} \in[0.9,1.0] \\
\mathrm{S}=0.9, \mathrm{~S}<0.9
\end{array}\right.
$$

From this we can see that the smaller the value, the more suitable it is within the scope of 0.9 to 1.0 , for which the waste temperature will be less, and the maize varieties mature in the shortest time under the conditions of required temperature.

\subsubsection{Lodging and diseases and pests membership function}

The evaluation of diseases and pests can be described of two aspects from the qualitative and quantitative. From the qualitative evaluation, four conditions exist as follows:

(1) A resistance of the corn varieties in a low stress environment promotion.

(2) A resistance of the corn varieties in a high stress environment to promote. 
(3) Not an anti-corn variety in a low stress environment promotion.

(4) A resistance of the corn varieties not in a high stress environment to promote.

While, from the quantitative evaluation, the relationship between varieties susceptible reliability and environmental stress will be researched and established. This paper attempted to quantitatively evaluate the impact of pest on yield. The formulas can be expressed as follows:

$$
\begin{aligned}
& \left\{\begin{array}{l}
K_{i}=\frac{\bar{D}_{i}-\hat{D}_{i}}{D_{\max }^{*}} \quad(\mathrm{i}=1,2 \ldots, 12) \quad R^{2} \neq 0 \\
K=-0.01 \text { or }-0.11 \quad R^{2}=0
\end{array}\right. \\
& \hat{D}_{i}=W_{1} * D_{i}^{*}+W_{2} * D_{i \max }^{*} \\
& D_{i}^{*}=a+b x \quad W_{1}=R^{2} \quad W_{2}=1-R^{2}
\end{aligned}
$$

In formula $5, \mathrm{x}$ is the stress degree of diseases and pests and lodging rate of each county, $D^{*}$ is stress degree of diseases and pests of some variety in different environment, $D_{i}^{*}$ is a linear function about environmental stress, $\mathrm{x}$, $D_{i}^{*}$ is a number belonging to $[0,9]$ or $[0,100]$ at random, $R^{2}$ is a correlation coefficient of the linear function.

In formula $4, D_{i}{ }^{*}$ which amended by the management pessimistic act, represents the forecast of degree of the maize varieties flu disease, a function about environmental stress as well. $D_{i_{\max }}{ }^{*}$ is the maximum value of a variety of lesions to the disease, Taking into account $R^{2}$ of $D_{i}{ }^{*}$ smaller, $\hat{D}_{i}$ is a method when the diseases and pests sensitivity and the degree of environmental stress linear fit is not so good. When $R^{2}$ small, only illustrates the extent of fitting not good, and lack of regularity, however, could not explain the resistance of the variety. Then pessimistic assumption $\hat{D}_{i}$ is mainly decided by the maximum value. On the opposite side, is mainly decided by $D_{i}{ }^{*} . W_{1}, W_{2}$ are the respectable weights of $D_{i}^{*}$ and $D_{i \max }^{*}$. When $R^{2}$ of $D_{i}^{*}$ larger, the weight of $D_{i}^{*}$ is larger, otherwise, smaller.

Formula 3 is on behalf of that diseases of a certain type of a variety increase or reduce production levels. $i$ is the type of the diseases and pests and lodging (such as big spot disease, lodging), $\bar{D}_{i}$ is an average value of a variety of disease extent, the average level of a variety in the study area susceptible also. If $\left(\bar{D}_{i}-\hat{D}_{i}\right)>0$, then it expressed the susceptible of the 
variety in the region less than the average value of the variety, which means the variety is more appropriate in the region to promote, and increases the production, vice versa.

\subsection{Model establish}

Suitability evaluation of new maize varieties is based on the average yield per acre under climatic conditions, lodging and diseases, pests in the end (Tong, 1997; Wang 2006). Therefore, we need to forecast the new varieties of maize yield per acre after establishing accumulated temperature, lodging and diseases and pests membership function, finally, ascertain the suitability evaluation model of new maize varieties.

\subsubsection{Method of forecasting new maize varieties average yield per acre}

The method is similar to the method of calculating diseases and pests susceptible. The formulas can be expressed as follows:

$$
\begin{aligned}
& \hat{C}_{i}=W_{1} * C_{i}^{*}+W_{2} * C_{i \min }^{*} \\
& C_{i}^{*}=a+b x \quad W_{1}=R^{2} \quad W_{2}=1-R^{2}
\end{aligned}
$$

Formula 7 is a linear function based on the environmental average yield per acre within east of north China trial regional and average yield per acre of a variety, $R^{2}$ is larger, the fitting of the linear equation is better. $\mathrm{x}$ is environmental average yield per acre in each county.

In formula $6, C_{i}^{*}$ which amended by the management pessimistic act, represents a forecast of the maize varieties average yield per acre in every counties, a function on environmental average yield per acre, $C_{i \min }^{*}$ is the minimum average yield. Pessimistic assumption that when $R^{2}$ smaller, that is the extent of fitting not well, the weight of the lowest average yield is larger. $W_{1}, W_{2}$ are the respectable weights of $C_{i}^{*}$ and $C_{i \min }^{*}$.

\subsubsection{Suitability evaluation model of new maize varieties}

According to the impact factors, the weights of each factors and the membership function, we can establish the evaluation model, which can be used to compare the appropriate level of a maize variety in every county, can be compared the suitable degree of new maize varieties in a particular 
county. The suitable degree is measured by average yield per acre. The higher the average yield, the better appropriate in the county, the better promotion. Comprehensive above formula, we can get the suitability evaluation model of new maize varieties, as shown below:

$$
\hat{Y}=\hat{C} *\left(1+T+\sum_{i=1}^{12} K_{i} * V_{i}\right) \quad(\mathrm{i}=1,2 \ldots 12)
$$

In formula $8, \hat{C}$ is the average yield per acre of a variety in one county by environmental average yield per acre of every counties in east of north China. Calculation is Similar to $K_{i}, \hat{Y}$ is average yield per acre forecasting by considering temperature, lodging, diseases and pests. Thus, we can judge a variety suitable for which counties and not by the level of forecasting value, meanwhile, the appropriate level can be judged as well. Different varieties comparison, the model can calculate the average yield per acre level, with a judgment in which the county more suitable.

\section{SUITABILITY EVALUATION}

Take the new variety 628412 and HAOYU12 for reference, we can judge which will be more appropriate in the east of north China through the suitability evaluation model of new maize varieties. Take the 628412 suitability evaluation as example:

\subsection{Introduction to 628412}

In 2005 , the production of the region test was $760.0 \mathrm{~kg}$ per acre; increasing $17.70 \%$ compared to NONGDA108, The effect was remarkable, occupied 1 st, 22 fields increased, and didn't have decreased fields.

The average growth period of the variety is 127 days, earlier 4 days than NONGDA108. The height of the adult plants is about 330 centimeters. The height of the tassel is 120 centimeters, and the length of the tassel is 19.8 centimeters, $16-18$ rows. The weight of the single tassel is $245 \mathrm{~g}$. The weight of one hundred corn kernels is $39.0 \mathrm{~g}$. It resists various diseases and pests through appraising. If diseases and pests appear in the fields, it resists all the plant diseases. 


\subsection{Process of 628412 evaluation}

\subsubsection{Calculation of the suitability of the accumulated temperature}

The accumulated temperature of growth period of 628412 requires $2434.2^{\circ} \mathrm{C}$. So we can get the suitability of the accumulated temperature of 628412 in the new maize varieties promotion fields in the 223 counties of four provinces in the east of north China according to the method of the suitability of the accumulated temperature. As shown in figure 1:

\begin{tabular}{|c|c|c|c|c|c|c|}
\hline \multicolumn{7}{|c|}{8 Mitrosofit Exatel - Book1 } \\
\hline 짐 & 文件 (E) 编轱 & 辑 (E) 视图 & 插入(I) & 工具 $(\mathrm{T})$ & 数据 (D) & 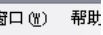 \\
\hline \multicolumn{7}{|c|}{ 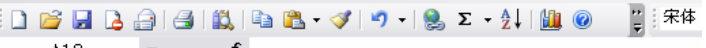 } \\
\hline & A18 & $-\quad t$ & $f_{x}$ & & & \\
\hline & A & B & C & D & $\mathrm{E}$ & $\mathrm{F}$ \\
\hline 1 & Counties & ID & Environmental & Growth & Result & Suitabi] \\
\hline 2 & ChangTu & 211224 & 3025.5 & 2434.2 & 0.8045612 & 0.9 \\
\hline 3 & HuaDian & 220282 & 3025.5 & 2434.2 & 0.8045612 & 0.9 \\
\hline 4 & XiFeng & 211223 & 2922.2 & 2434.2 & 0.8330025 & 0.9 \\
\hline 5 & KangPing & 210123 & 3094.4 & 2434.2 & 0.7866468 & 0.9 \\
\hline 6 & KaiYuan & 211282 & 3010.8 & 2434.2 & 0.8084894 & 0.9 \\
\hline 7 & ZhangWu & 210922 & 3149.9 & 2434.2 & 0.7727864 & 0.9 \\
\hline 8 & $\mathrm{FaKu}$ & 210124 & 3129.7 & 2434.2 & 0.7777742 & 0.9 \\
\hline 9 & WeiChang & 130828 & 2557.9 & 2434.2 & 0.95164 & 0.95 \\
\hline 10 & FuXin & 210921 & 3217.9 & 2434.2 & 0.7564561 & 0.9 \\
\hline 11 & TieFa & 211281 & 3108.8 & 2434.2 & 0.7830031 & 0.9 \\
\hline 12 & TieLing & 211221 & 3088.5 & 2434.2 & 0.7881496 & 0.9 \\
\hline 13 & QingYuan & 210423 & 2791.5 & 2434.2 & 0.8720043 & 0.9 \\
\hline 14 & TianPing & 211322 & 3184.1 & 2434.2 & 0.764486 & 0.9 \\
\hline
\end{tabular}

Figure 1. The accumulated temperature of 628412 in the 223 counties

According to the rank of the suitability of the accumulated temperature and the suitability of the 223 counties, 223 counties will be divided into two categories of appropriate and inappropriate, as shown in figure 2:

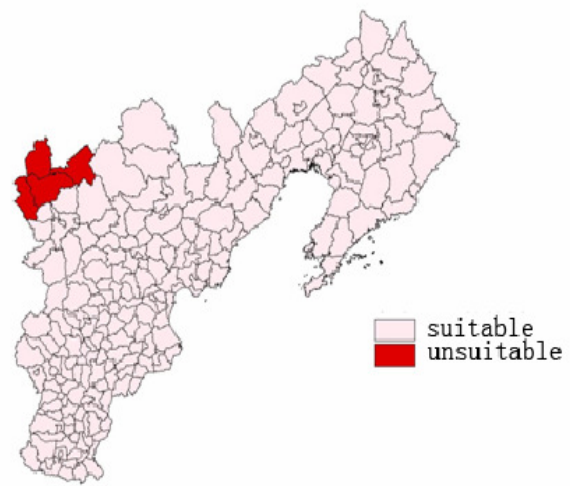

Figure 2. The map of accumulated temperature suitable counties of 628412 in the promotion fields (four provinces) in the east of north China 
We can know from the figure, in the new maize varieties promotion regions of the east of north China, 5 counties are inappropriate. The other 218 counties are appropriate for promoting 628412. The inappropriate counties are Kang Bao County, Zhang Bei County, Shang Yi County, Gu Yuan County, and Ping Shan County. The data of inappropriate degree of these counties as shown in Table 3:

Table 3. 628412 inappropriate counties in the promotion fields in the east of north China

\begin{tabular}{lccccc}
\hline County & Kang Bao & Zhang Bei & Shang Yi & Gu Yuan & Ping Shan \\
\hline Appropriate of accumulated temperature & 1.12 & 1.04 & 1.09 & 1.04 & 1.04 \\
\hline
\end{tabular}

\subsubsection{Yield forecasting}

The scatter gram on average yield per acre in trial areas of varieties and environmental average yield per acre is shown in figure 3 .

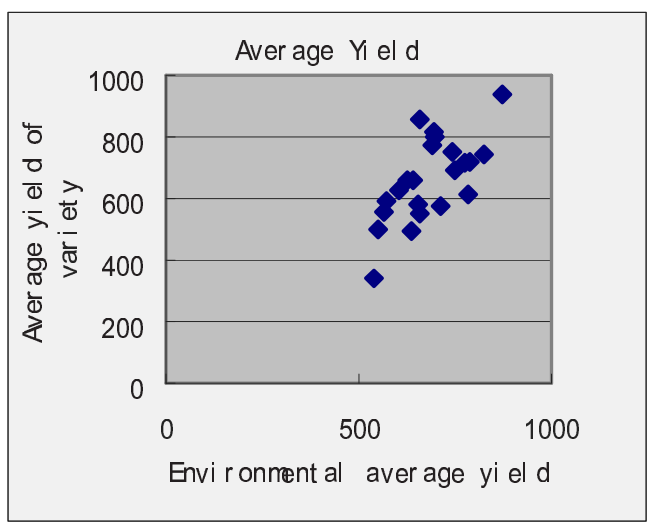

Figure 3. The relationship between 628412 average yield and environmental average yield

From figure 3, we can see the dots distribution showing a certain linear. According this, we can get the linear equation on varieties average yield per acre and environmental average yield per acre.

$$
\begin{aligned}
& C^{*}=-37.462+1.023 x \quad R^{2}=0.462 \\
& \hat{C}=0.462 *(-37.462+1.023 x)+0.538 * 341.1
\end{aligned}
$$

\subsubsection{Big spot disease}

(1) $D_{i}^{*}$ linear equation fitness and $R^{2}$ solution

The scatter gram about the degree of the sensitivity of big spot disease in trial areas and environmental stress on big spot disease is shown in figure 4. 


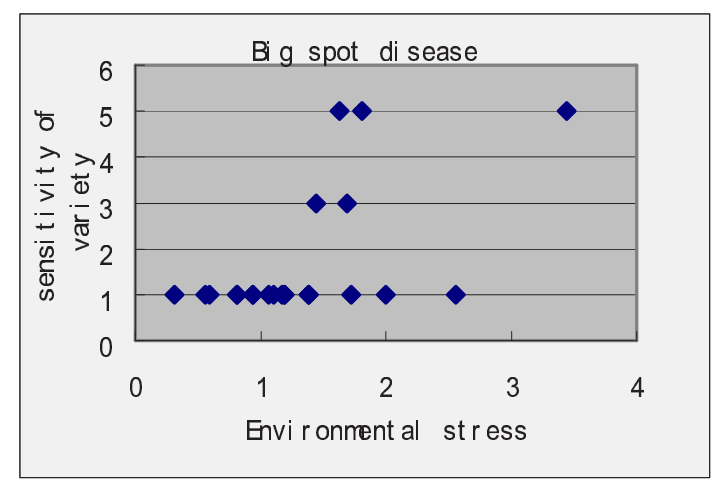

Figure 4. The relationship between 628412 sensitivity of big spot disease and environmental stress

From figure 4, we can see the dots distribution showing scatter, $R^{2}$ will be smaller. According this, we can get the linear equation on the degree of the sensitivity of big spot disease in trial areas and environmental stress on big spot disease.

$$
D_{1}^{*}=0.081+1.177 x \quad R^{2}=0.332
$$

(2) $\hat{D}_{i}$ calculation amended by $D_{i}^{*}$ using pessimistic assumptions

By fitness equation $D_{1}{ }^{*}=0.081+1.177 x$ and environmental stress on big spot disease in 223 counties of east of north China, we can calculate the sensitivity of big spot disease, get the maximum 5 as $D_{i \max }^{*}$, Thus:

$$
\hat{D}_{1}=0.332 *(0.081+1.177 x)+0.668 * 5
$$

For $\hat{D}_{1}$, it is the forecasting value of the degree of the sensitivity of big spot disease in 223 counties under pessimistic assumptions.

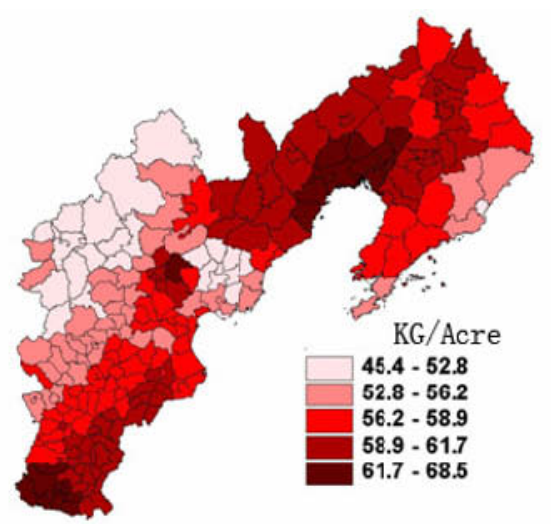

Figure 5. The impact of big spot on yield of 628412 
(3) K calculation

We can get the impact of pest on yield by taking the average 1.861 as $\bar{D}_{1}$

$$
K_{1}=\frac{1.186-\hat{D}_{1}}{9}
$$

Similarly, we can calculate the impact of evaluation factors on yield for small spot disease, bending fungus, gray leaf spot disease, black silk, smut, stem rot, sheath blight, MDM, rough dwarf virus, corn borer and lodging rate.

\subsection{Calculation by model}

According to the formula 8, putting the output of 628412 variety evaluation process into evaluation model, it may be:

$$
\hat{Y}=\hat{C} *\left(\begin{array}{l}
(1-s) * 1+\frac{1.186-\hat{D}_{1}}{9} * 0.5+\frac{2.097-\hat{D}_{2}}{9} * 0.6+\frac{1.179-\hat{D}_{3}}{9} * 0.6+\frac{1.132-\hat{D}_{4}}{9} \\
* 0.5+\frac{1.441-\hat{D}_{5}}{100} * 0.7-0.01 * 0.3+\frac{2.615-\hat{D}_{7}}{100} * 0.5+\frac{1.381-\hat{D}_{8}}{9} * 0.2-0.111 * 0.1 \\
+\frac{1.333-\hat{D}_{10}}{100} * 0.5+\frac{1.514-\hat{D}_{11}}{100} * 0.2+\frac{3.788-\hat{D}_{12}}{100} * 0.4+1
\end{array}\right) .
$$

There are 218 counties meeting temperature, so the calculated only carries on 218 counties. We can forecast the average yield per acre under the influence of the weather, pests, lodging integrated in every county, as shown

\begin{tabular}{|c|c|c|c|}
\hline \multicolumn{4}{|c|}{ 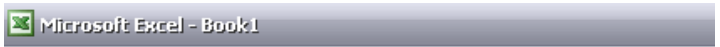 } \\
\hline 짐 & 文件 (B) 编阵 & 视图 & 插入(I) 格式 (0) \\
\hline \multicolumn{4}{|c|}{ 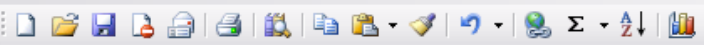 } \\
\hline & A20 & $\Rightarrow \quad f$ & $f_{x}$ \\
\hline & A & B & $\mathrm{C}$ \\
\hline 1 & Counties & ID & Forecasted yield(KG/Acre) \\
\hline 2 & ChangTu & 211224 & 253 \\
\hline 3 & HuaDian & 220282 & 253 \\
\hline 4 & XiFeng & 211223 & 250.9 \\
\hline & KangPing & 210123 & 256 \\
\hline 6 & KaiYuan & 211282 & 264.9 \\
\hline 7 & ZhangWu & 210922 & 249.9 \\
\hline 8 & $\mathrm{FaKu}$ & 210124 & 263.1 \\
\hline 9 & WeiChang & 130828 & 234.7 \\
\hline 10 & FuXin & 210921 & 239.5 \\
\hline 11 & $\mathrm{TieFa}$ & 211281 & 271.7 \\
\hline 12 & TieLing & 211221 & 282.5 \\
\hline 13 & QingYuan & 210423 & 257 \\
\hline & & 211322 & \\
\hline
\end{tabular}
in figure 6 :

Figure 6. The average yield per acre forecasted of 628412 
The higher average yield per acre forecasted the more suitable for 628412 promotions in this county. In the results map of suitability evaluation of 628412, we use different legend shapes and colors to distinguish the fitness level among them.

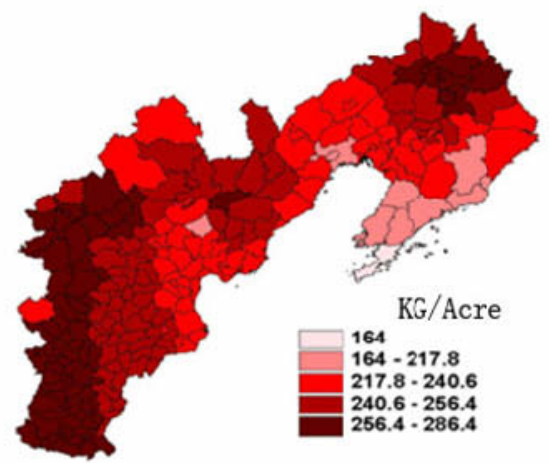

Figure 7. Distribution of average yield per acre forecasted of 628412 in suitable counties

In the same way, HAOYU12 is assessed. We can obtain the formula:

$$
\hat{Y}=\hat{C} *\left(\begin{array}{l}
(1-s) * 1+\frac{1.518-\hat{D}_{2}}{9} * 0.5+\frac{1.193-\hat{D}_{2}}{9} * 0.6+\frac{1.721-\hat{D}_{2}}{9} * 0.6+\frac{1.185-\hat{D}_{2}}{9} \\
* 0.5+\frac{1.282-\hat{D}_{2}}{100} * 0.7-0.01 * 0.3+\frac{1.363-\hat{D}_{7}}{100} * 0.5+\frac{1.570-\hat{D}_{8}}{9} * 0.2-0.111 * 0.1 \\
+\frac{1.499-\hat{D}_{10}}{100} * 0.5+\frac{1.517-\hat{D}_{11} * 0.2+\frac{3.780-\hat{D}_{12}}{100} * 0.4+1}{100} * 2
\end{array}\right) .
$$

\begin{tabular}{|c|c|c|c|}
\hline \multicolumn{4}{|c|}{ 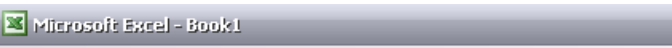 } \\
\hline \multirow{2}{*}{\multicolumn{4}{|c|}{ 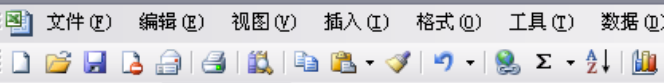 }} \\
\hline & & & \\
\hline \multicolumn{4}{|c|}{$\mathrm{A} 17 \quad \mathrm{f} \quad f_{x}$} \\
\hline & A & B & $\mathrm{C}$ \\
\hline 1 & Counties & ID & Forecasted yield(KG/Acre) \\
\hline 2 & ChangTu & 211224 & 355.6 \\
\hline 3 & HuaDian & 220282 & 355.6 \\
\hline 4 & XiFeng & 211223 & 347.3 \\
\hline 5 & KangPing & 210123 & 360.5 \\
\hline 6 & KaiYuan & 211282 & 375.6 \\
\hline 7 & ZhangWu & 210922 & 356.8 \\
\hline 8 & $\mathrm{FaKu}$ & 210124 & 376.9 \\
\hline 9 & WeiChang & 130828 & 340.2 \\
\hline 10 & FuXin & 210921 & 388.9 \\
\hline 11 & TieFa & 211281 & 414.3 \\
\hline 12 & TieLing & 211221 & 339.5 \\
\hline 13 & QingYuan & 210423 & 351.5 \\
\hline 14 & TianPing & 211322 & 425.3 \\
\hline
\end{tabular}

Figure 8. The average yield per acre forecasted of HAOYU12 


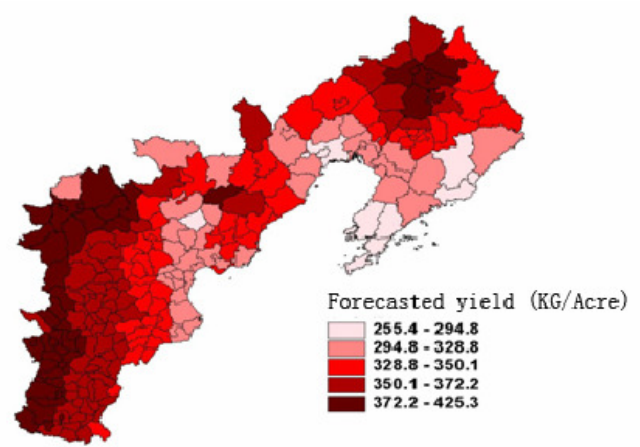

Figure 9. Distribution of average yield per acre forecasted of HAOYU12 in suitable counties

\section{CONCLUSIONS}

We can draw the following conclusions after the suitability evaluation of 628412 and HAOYU12:

(1) The average yield per acre forecasted of HAOYU12 is higher than 628412 in appropriate counties. It shows HAOYU12 is more suitable for promotion than 628412 in most parts of the east of north China.

(2) Lodging, big spot disease, small spot disease, gray leaf spot disease, sheath blight, bending fungus have greater impact on corn yield in east of north China. In other words, varieties of maize resisting the above diseases should be more suitable for promotion in the east of north China.

(3) The introduction of management of pessimistic assumptions to suitability evaluation model of new maize varieties avoids undervaluing a variety of inappropriate, provides a reliable guarantee to promote the new maize variety.

(4) The suitability evaluation model of new maize varieties can solve the traditional mode of promoted region choosing and make up for the deficiencies in agricultural expert system, assist in raising the accuracy and scientific choice. It is of great practical significance to new maize varieties promotion evaluation from the qualitative evaluation and experience to the quantitative model.

\section{ACKNOWLEDGEMENTS}

This research is funded by the Support of Science and Technology Project of the State, Programmed award No. 2006BAD10A01 from March 2007 to November 2009. 


\section{REFERENCES}

HuangHe, Suitability evaluation of regional vegetable field by GIS, Fujian journal of agricultural sciences, 2004, 19(2): 108-112

LiaoQin, SunShixian, New maize varieties dynamic of China, China agricultural science and technology press. 2002

LiHong, SunDanfeng, ZhangFengrong, ZhouLiand. Suitability evaluation of fruit trees in Beijing Western Mountain areas based on DEM and GIS. Transactions of the CSAE, 2002, 18(5): $250-255$

LiuMingChun, LiQiaozhen, YangXiaoli. Research on maize climate suitable for ecological of Gansu province, Agricultural research in dry areas, 2003, 23(3): 112-117

TongPinya, ChenYannian, Corn growth and Yield model. China agricultural science and technology press. 1997

WangBaotang, Skills on extending maize new variety, China seed industry. 2006(3):17-18

WangYongguang, AiWanxiu. Analysis and forecast of $\geq 10 \square$ effective accumulated temperature in Northeast China, China agricultural meteorology, 1997, 3(18): 39-44

YuRonghuan, ShunMengmei, ZhangLijuan. The re-division about the quantity of heat resources and the accumulated temperature zone of Heilongjiang province, Natural sciences journal of Harbin normal university, 1997, 3(13): 98-102

ZhangJing, FengJinxia, BianXinmin, ZangMin. Variable weight approach in evaluation of corps ecological adaptability, Journal of Nanjing Agricultural University, 2006, 29(1): 13-17 